\title{
Arts en conflit : chanson et roman en Allemagne de Thomas Mann à Wolfgang Koeppen
}

\section{François Genton}

\section{(2) OpenEdition}

\section{Journals}

\section{Édition électronique}

URL : http://journals.openedition.org/recherchestravaux/458

DOI : 10.4000/recherchestravaux.458

ISSN : 1969-6434

Éditeur

UGA Éditions/Université Grenoble Alpes

\section{Édition imprimée}

Date de publication : 15 mai 2011

Pagination : 153-168

ISBN : 978-2-84310-200-4

ISSN : 0151-1874

\section{Référence électronique}

François Genton, «Arts en conflit : chanson et roman en Allemagne de Thomas Mann à Wolfgang Koeppen », Recherches \& Travaux [En ligne], 78 | 2011, mis en ligne le 15 novembre 2012, consulté le 07 septembre 2020. URL : http://journals.openedition.org/recherchestravaux/458 ; DOI : https://doi.org/ 10.4000/recherchestravaux.458 


\section{Arts en conflit : chanson et roman en Allemagne de Thomas Mann à Wolfgang Koeppen}

Au XIX siècle, la France, le Royaume Uni et la Russie produisent des romanciers universels tandis que l'Allemagne, où se publient nombre de romans, brille surtout par ses grands musiciens. Sur le plan intermédial, on note une forte présence internationale de la poésie et du drame allemands en musique, mais une moindre représentation du genre narratif en général et du roman en particulier. On retiendra ici surtout les romans que Goethe a publiés au XviII ${ }^{\mathrm{e}}$ siècle, Les Souffrances du jeune Werther (1774) et Les Années d'apprentissage de Wilhelm Meister (1796), avec son personnage au plus au point musical de Mignon. Richard Wagner, à la fois compositeur et auteur, jouit à la fin du siècle d'une renommée universelle, confirmant la représentation d'une musicalité essentielle de l'Allemagne. Ses œuvres inspirées de la mythologie germanique confirment l'idée d'une Allemagne profonde, intérieure, tourmentée, mais aussi antique et puissante. Comment le roman allemand moderne, qui s'impose sur le plan international avec Les Buddenbrooks. Déclin d'une famille (Buddenbrooks. Verfall einer Familie', I90I) de Thomas Mann, a-t-il pris en compte cette prééminence nationale et internationale de la musique allemande sur les autres arts, a fortiori sur le roman, genre pourtant particulièrement brillant aux frontières orientale et occidentale de l'Europe centrale? Comment a-t-il affirmé sa légitimité de medium artistique susceptible de dire la vie moderne? Cette étude s'intéressera ici également au

I. Th. Mann, Buddenbrooks. Verfall einer Familie, Francfort, Fischer, 2002. Nous citerons la version brève du titre. La traduction de G. Bianquis (1932) a été rééditée en 1992 par Fayard. Dans cette étude, nous avons traduit toutes les citations allemandes. 
roman Le Sujet de l'Empereur (Der Untertan², I9I4-I9I8) d'Heinrich Mann, le frère aîné de l'auteur des Buddenbrooks. Comment les relations entre un genre romanesque en plein essor et la musique ont-elles évolué, parallèlement à l'émergence d'un marché grandissant de la musique populaire, jusqu’à l'avant-garde artistique de la République de Weimar? Dans ce contexte, nous étudierons Berlin Alexanderplatz ${ }^{3}$ d'Alfred Döblin (I929). Après avoir tenté de répondre à ces questions, cette étude se concentrera sur un roman particulièrement représentatif d'une volonté affichée au sortir de la glaciation hitlérienne de renouer avec l'avant-garde allemande et européenne d'avant 1933. La Serre (Das Treibhaus, 1953 ${ }^{4}$ de Wolfgang Koeppen est d'une certaine façon une tentative de rattraper le temps perdu et de regagner au roman tout le potentiel de modernité dont il a été privé en Allemagne durant les interminables douze années du Troisième Reich : cette modernité retrouvée parvient-elle, dans le domaine intermédial roman-musique, à s'imposer sans que ne subsiste aucune trace de la concurrence et, éventuellement, des conflits d'antan entre musique et roman'?

La nation allemande s'est d'une certaine façon construite par le chant, des chants religieux du Moyen Âge aux chorals luthériens, des Carmina Burana au Livre du chant de taverne (Kommersbuch) des corporations étudiantes de la seconde moitié du XIX ${ }^{\mathrm{e}}$ siècle, du chant populaire $\left(\right.$ Volkslied $\left.^{6}\right)$ redécouvert

2. H. Mann, Der Untertan, Francfort, 2007. Le Sujet de l'Empereur, traduction de P. Budry (I928 sous le titre Sujet!), révisée par D. Saadtjan et rééditée en 1982 par les Presses d'aujourd'hui.

3. A. Döblin, Berlin Alexanderplatz, Munich, dtv, 1979. D'abord disponible dans une transposition de Z. Motchane (Berlin Alexanderplatz, I933), le roman vient d'être retraduit par O. Le Lay, Gallimard, 2009.

4. W. Koeppen, Das Treibhaus [1953], Francfort, Fischer, 1972. Ce roman n'a pas été traduit en français. Nous n'avons pas pu consulter l'édition critique élaborée par U. Treichel et qui doit paraître en octobre 20Io, comme t. 5 des œuvres en I6 vol. (Francfort, Suhrkamp).

5. Ce roman représente mieux l'Allemagne d'après 1945 que Doktor Faustus (1946) de Th. Mann, qui est une somme que l'on peut juger, d'un point de vue esthétique, bien supérieure au roman de Koeppen. Th. Mann y pense l'aventurisme national allemand à partir du destin d'un compositeur, Adrian Leverkühn, et du thème faustien du pacte avec le diable. Les relations "intermédiales» entre chanson et roman sont un sujet neuf. En Allemagne, on s'intéresse traditionnellement à celles qui relient la musique "classique» et les romanciers. Ainsi, Fr. Ehinger (Gesang und Stimme im Erzählwerk von Gottfried Keller, Eduard von Keyserling und Thomas Mann Würzburg, Königshausen \& Neumann 2004), étudiant l'utilisation du chant dans le roman "réaliste», a-t-elle mis l'accent sur les discordances et une certaine disharmonie à l'ére industrielle. En ce qui concerne La Serre, on notera que la critique (par exemple K. H. Götze, Wolfgang Koeppens "Das Treibhaus», Munich, Fink, 1985) défend la thèse d'une critique pertinente de la "société bourgeoise» et ne s'intéresse pas à la chanson.

6. Ce dernier terme a été forgé par J. G. Herder en 1773 dans un texte intitulé «Extrait d'une correspondance sur Ossian et les chants des peuples anciens» (Auszug aus einem Briefwechsel über Oßian und die Lieder alter Völker) dans De la manière et de l'art des Allemands 
à l'époque du Sturm und Drang par Herder aux innombrables recueils et à la multiplication des chorales au XIX ${ }^{\mathrm{e}}$ siècle, de la pratique intensive du Lied (en allemand : Kunstlied, chant d'art) dans les élites aristocratique et bourgeoise à la généralisation d'une sociabilité "chantante" à quasiment tous les secteurs de la société. Au xx siècle on constate en Allemagne, comme partout ailleurs, le passage au chant "reproduit», diffusé et amplifié par des moyens techniques. Le couple relativement harmonieux que formaient le chant populaire et le chant d'auteur, dont témoignent les nombreuses adaptations populaires de poèmes, voire de Lieder à l'usage du peuple, disparaît au profit de l'émergence d'une industrie de l'opérette et de la chanson moderne (Schlager) qui masque, en Autriche et en Allemagne, les tensions qui divisent la société et qui s'expriment, elles aussi, par des hymnes. L'Allemagne démocratisée d'après 1945, si elle découvre peu à peu l'univers de la musique "de masse" anglo-saxonne, conserve jusqu'à aujourd'hui cet attachement au chant en groupe et aux occasions de le pratiquer, de l'église au stade de football, des chapiteaux du carnaval et des fêtes foraines aux salles, aux chapelles et aux églises où se produisent les chorales de l'école, du village ou du quartier. En 1966 Franz Beckenbauer et les footballeurs de l'équipe nationale et sept ans plus tard le président de la République fédérale d'Allemagne Walter Scheel ont vendu des millions de disques ${ }^{7}$ !

Le genre épique ne pouvait que rendre compte de cette omniprésence du chant. Récit, poésie et chant, une solide tradition allemande créée par Goethe et développée par les romantiques associe ces trois mots. Les poésies des Années d'apprentissage de Wilhelm Meister (Wilhelm Meisters Lehrjahre, I795-1796) ont inspiré des dizaines de compositeurs dans toute l'Europe, à commencer par le poème dans lequel Mignon évoque la nostalgie de l'Italie, le pays où «fleurissent les citronniers». Les nouvelles allemandes du XIx ${ }^{\mathrm{e}}$ siècle fourmillent de poésies bientôt mises en musique, par exemple "Celui à qui Dieu manifeste sa faveur" (Wem Gott will rechte Gunst erweisen ${ }^{8}$ ), poésie du premier chapitre de Mémoires d'un propre à rien (Aus dem Leben eines Taugenichts, I826), nouvelle du Silésien Joseph von Eichendorff. Cette spécificité poétique et musicale du genre narratif allemand est peut-être l'un des éléments qui expliquent son relatif manque de retentissement international à

(Herder - Goethe - Frisi - Möser, Von deutscher Art und Kunst, Stuttgart, Reclam, 1968, p. 24), le manifeste le plus connu du Sturm und Drang.

7. Ces "artistes» ont interprété respectivement Personne ne peut séparer les bons amis (Gute Freunde kann niemand trennen), une chanson "de variété» fabriquée pour l'occasion, et Perchés sur la voiture jaune (Hoch auf dem gelben Wagen), une chanson du XIX siècle, proche de la variété.

8. Le poème fut mis en musique en I833 par Friedrich Th. Fröhlich. 
l'heure de la naissance en Europe de la grande tradition «réaliste» en France, en Angleterre et en Russie de récits longs et brefs attachés aux questions du monde moderne. Malgré la relative renommée des Années d'apprentissage de Wilhelm Meister, pour lequel le germaniste hégélien Karl Morgenstern forge en I819 le concept de Bildungsroman, et le succès en France des contes d'Hoffmann, le genre narratif allemand et plus particulièrement le roman restent méconnus sur le plan international. Cette thèse se vérifie particulièrement en ce qui concerne les premiers romanciers « réalistes» de langue allemande, qu'il s'agisse du Suisse Gottfried Keller et de son roman Henri le Vert (Der grüne Heinrich, I853-I890) ou de Theodor Fontane, dont on considère l'œuvre romanesque, qui paraît dans les dernières décennies du XIX ${ }^{\mathrm{e}}$ siècle, comme fondatrice. Il est vrai que même dans l'Allemagne de cette époque le roman ne compte pas. En I883, dans son histoire de la littérature allemande, le germaniste Wilhelm Scherer (I84I-I886), qui vient d'inaugurer la chaire de littérature allemande moderne à l'université Friedrich-Wilhelm de Berlin, ne consacre qu'une vingtaine de pages sur 83I à un chapitre intitulé "Récits» (Erzählungen). On y parle surtout de Goethe et de Jean Paul, c'est-à-dire d'auteurs qui ont disparu depuis plus d'un demi-siècle, pour conclure à une prééminence de la nouvelle sur le roman".

Hegel et Lukács ont défini à un siècle de distance le roman comme une "épopée bourgeoise moderne" (eine moderne bürgerliche Epopöe ${ }^{\mathrm{10}}$ ) ou comme l'«épopée d'un monde abandonné par Dieu» (Epopöe einer gottverlassenen

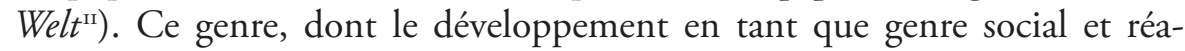
liste s'accélère après la chute de l'Ancien Régime et la révolution industrielle, a rencontré en Allemagne deux obstacles. D'abord l'absence d'une nation organisée autour d'une grande capitale économique et politique. Les Allemands - et même leurs élites - ne sont pas habitués à coexister dans l'univers "totalisant» que demande le roman moderne. Le second obstacle est lié à la structure sociale et mentale de l'Empire fondé en i87i. Malgré un essor économique qui fait du pays la première puissance européenne à la veille de la Première Guerre mondiale, l'Allemagne ne se modernise pas de la même manière que ses grands voisins occidentaux et parvient à adapter, en les conservant, les traits essentiels de l'Ancien Régime à la réalité économique, technique et politique du pays. C'est une combinaison de fidélité dynastique et d'adhésion à la monarchie de droit divin d'un côté et de supériorité

9. W. Scherer, Geschichte der deutschen Literatur, Berlin, Th. Knaur, s.d., p. 725-745.

Io. G. Fr. Hegel, «Vorlesung über die Ästhetik» (cours tenus entre I818 et I828), cité par H. Steinecke et Fr. Wahrenburg (éd.), Romantheorie. Texte vom Barock bis zur Gegenwart, Stuttgart, Reclam, 1999, p. 297.

II. G. Lukács, «Die Theorie des Romans» (I916), ibid., p. 4II. 
nationale «ethnique» de l'autre qui est au principe du nouvel État. Le creuset de la "communauté» nationale, c'est l'armée, encadrée par des officiers issus de l'aristocratie, et une école et une élite étudiante qui en singent le ton et les méthodes. La mémoire des grands soldats, d'Arminius à Frédéric le Grand, domine celle des grands poètes ou, plutôt, on tente de mobiliser ces derniers pour la cause des Hohenzollern : les auteurs auront eu le mérite d'anticiper par l'unité culturelle de la nation l'unité politique qui fut l'œuvre militaire de la dynastie prussienne. L'Allemagne s'intéresse de près au grand roman européen, mais le genre semble "exotique» dans une nation qui commence juste à se fédérer et qui est par ailleurs peu disposée à assumer, même dans ses fictions, les divisions sociales qui constituent l'un des principaux sujets, sinon le principal, du roman moderne. L'Empire triomphant des Hohenzollern ne se vit en totalité que sur un mode imaginaire, et dans un passage clé du roman de Heinrich Mann Le Sujet de l'Empereur (Der Untertan, I9I4-I9I8), le genre romanesque est renvoyé à ses origines non allemandes, voire anti-allemandes, quand Lohengrin de Wagner apparaît comme le sommet de ce que l'art allemand peut produire, selon les conclusions que tire Diederich, le «héros» du roman, devant son épouse :

"Voilà l'art qu'il nous faut!» s'écria Diederich. «Voilà un art allemand!» Il lui semblait en effet que le texte et la musique remplissaient tous les critères nationaux. La révolte était pareille au crime, l'ordre établi, légitime était célébré avec faste, on attachait le plus grand prix à l'aristocratie et à la monarchie de droit divin et le peuple, un chœur éternellement surpris par les événements, se battait sans rechigner contre les ennemis de son maître. La base guerrière, le sommet mystique, tout y était. Le fait que l'homme représentât le sexe le plus beau et le plus aimé n'était pas le moindre aspect familier et sympathique de cette création. "Je sens mon cœur chavirer quand je vois cet heureux homme" chantaient les hommes et même le roi. Ainsi la musique elle-même participait-elle de ce bonheur viril, héroïque dans sa luxuriance, sachant rester impériale lorsqu'elle exprimait le désir. Qui eût pu résister? Il suffisait de représenter mille fois cet opéra pour que la cause nationale fit l'unanimité! Diederich le dit avec ces mots : "Le théâtre, voilà aussi l'une de mes armes». [...] Il proposa d'envoyer un télégramme de soutien à Wagner. Guste lui expliqua que c'était peine perdue. De la hauteur où l'avait conduit l'envol de ses pensées Diederich se mit à parler de l'art en général. Il y avait une hiérarchie des arts. "L'art suprême, c'est la musique, c'est pourquoi c'est l'art allemand par excellence. La musique est suivie du drame.»

«Pourquoi?» demanda Guste.

"Parce qu'on peut mettre parfois le drame en musique et qu'il n'est pas nécessaire de le lire, entre autres.»

«Et qu'est-ce qui vient après?»

"L'art du portrait bien sûr, à cause des portraits de l'empereur. Le reste n'a guère d'importance."

«Et le roman?» 
«Ce n'est pas un art. Du moins, Dieu soit loué, pas un art allemand : son seul nom l'indique ${ }^{12}$."

Rien d'étonnant donc à ce que les romans de l'ère wilhelminienne que la postérité a retenus aient traité la musique de Wagner, dans ses conditions réelles d'actualisation ${ }^{13}$. Le wagnérisme, dans les Buddenbrooks, précipite le déclin d'une famille de négociants hanséatiques. C'est Wagner qui réunit le sensible et peu fringant lieutenant René Maria (!) von Trotha et Gerda, l'épouse plutôt rigide de Thomas Buddenbrook. Le changement n'échappe pas à son mari qui se tourne vers Schopenhauer et se met à désirer la mort en sanglotant. Comme une sirène, Gerda "attira vers soi ${ }^{14}$ », Pfühl, l'organiste de cette ville luthérienne. Cette mère encourage aussi le wagnérisme de Johann (Hanno), l'enfant unique, qu'elle autorise à assister à une représentation de Lohengrin. La beauté de l'opéra consume «le courage et la force d'affronter la vie commune» d'un enfant, déjà affaibli par de nombreuses extractions de dent, comme une force intime, présente en lui dès le début de sa vie, et qui l'étouffait lentement ${ }^{15}$. Wagner n'est ainsi que le versant musical de la philosophie mortifere de Schopenhauer. Ce rappel du concert ouvre un chapitre cauchemardesque consacré à la journée de Hanno à l'école que dirige Wulicke, l'inexorable et inhumain directeur prussien que Kai, l'ami de Hanno, a affublé du surnom de "bon Dieu» (der liebe Gott). Le luthéranisme tolérant et bienveillant de Lübeck (la ville n'est jamais nommée dans le roman) est supplanté, après l'unité allemande, par une version plus militaire et monarchique du protestantisme. Le matin, on entonne à l'école le «Choral de Leuthen ", c'est-à-dire par «Rendez grâce à Dieu» (Nun danket alle Gott' ${ }^{16}$ ), un chant écrit et mis en musique en I630 par Martin Rinckart et arrangé par de nombreux compositeurs allemands, dont Jean-Sébastien Bach. Ce chant est aussi un hymne national depuis qu'on raconte que les soldats de Frédéric II de Prusse l'auraient "spontanément» entonné le 5 décembre 1757 au soir d'une grande victoire contre les Autrichiens. Déjà lors de la fête du

I2. H. Mann, Der Untertan, p. 354 et suiv.

13. Nous laissons de côté la nouvelle "Les enfants de Wotan»(Wälsungenblut), dans laquelle Th. Mann trace un parallèle entre le frère et la sœur d'une famille de la bourgeoisie judéo-allemande et le couple incestueux qu'a inventé Richard Wagner dans La Walkyrie. La nouvelle, écrite durant l'été 1905, ne parut qu'en I92I, avec une fin modifiée et en tirage limité. La belle-famille de l'écrivain s'était sentie visée, à bon droit. Dans la version de 1905, le frère, après l'inceste, répondait en yiddish à sa sœur qui s'interrogeait au sujet de son fiancé, un terne fonctionnaire : "Nun, was wird mit ihm sein? Beganeft haben wir ihn - den Goy." ("Et alors, qu'en sera-t-il de lui? Nous l'avons bien eu, ce goy.»).

I4. "Sie zog ihn zu sich herüber...", Th. Mann, op. cit., p. 499.

I5. Ibid., p. 70 I et suiv.

I6. Ibid., p. 706. 
centenaire de la firme Buddenbrook, ce même chant avait retenti ${ }^{17}$ : en juillet I868, Lübeck est entré dans la Confédération de l'Allemagne du Nord, dont le roi de Prusse, Guillaume I ${ }^{\text {er }}$, est le président, État qui préfigure l'Empire proclamé en I87I à Versailles. La militarisation de la vie et des institutions va de pair d'une part avec celle de la musique (le "choral de Leuthen») et d'autre part avec différentes formes illusoires de compensation, soit Wagner, soit le sectarisme protestant. Dès I856, la mère de Thomas Buddenbrook chante en effet un cantique particulièrement grotesque: "Je suis une charogne pour $\operatorname{corbeaux}^{18}[\ldots] »$. Christian, le frère dégénéré de Thomas, en dérision les chants de Noël et chante O Tantebaum! au lieu d'O Tannenbaum ${ }^{19}$ !, remplaçant le vénérable sapin (Tanne) par le mot «tante». L’aïeul Johann fredonnait sans talent ni prétention des chants gais et se délectait, à la fin de repas bien arrosés, de poésies libertines "traduites du français». Cet univers musical désuet est remplacé par un système plus complexe où voisinent la dureté du dressage et de la sélection et la nécessaire compensation, soit le wagnérisme mortifère, soit la stupidité des variétés modernes.

On a défini Le Sujet de l'Empereur comme une variation (Kontrafaktur) inspirée des Buddenbrooks, une intuition que confirme aussi une étude des motifs musicaux ${ }^{20}$. Dans le roman d'Heinrich Mann, Lohengrin fait aussi l'objet d'un long développement. Ici, l'opéra de Wagner confirme les pires préjugés nationalistes, antisémites et sexistes d'un sujet wilhelminien. Heinrich Mann, qui se documentait avec soin, avait assisté à Augsbourg à une représentation de cet opéra : "Que de bêtise dans un tel héros wagnérien, dans le chœur, partout ${ }^{21}$ !" En réalité l'Allemagne wilhelminienne de son roman est aussi parfaitement antimusicale que celle qu'annonce le Lübeck en voie de prussianisation de la fin des Buddenbrooks. Dans ce roman, dont l'action se déroule durant la dernière décennie du XIX ${ }^{e}$ siècle, soit vingt ans après la fin du roman de Thomas Mann, l'univers chansonnier de la nouvelle bourgeoisie nationaliste est un

17. Ibid., p. 490.

18. "Ich bin ein rechtes Rabenaas...", ibid., p. 278. Thomas Mann s'est inspiré d'un pastiche sans doute composé en 1840 par Friedrich Wilhelm Wolff. Voir Th. Mann, Große kommentierte Frankfurter Ausgabe, t. I.2, "Buddenbrooks. Verfall einer Familie. Kommentar von Eckhard Heftrich und Stephan Stachorski unter Mitarbeit von Herbert Lehnert », Francfort, Fischer, 2002, p. 3II.

19. Ibid., p. 535 .

20. Voir H. Koopmann, «Heinrich Mann : Der Untertan oder die Begründung der Macht aus dem Geist des Katechismus", Heinrich-Mann-Jahrbuch Io/1992, p. 90 et notre article: "Der Untertan, satire de Buddenbrooks ou correctif satrique?», dans M. EnderleRistori (dir.), "Der Untertan de Heinrich Mann. Pour un roman et une société modernes ", Cahiers d'histoire culturelle, université François-Rabelais, Tours, n² 2I, 2009, p. 47-63.

2I. "Wieviel Dummheit in so einem Wagner-Helden, in dem Chor, in allem!" Cité par St. Ringel, Heinrich Mann - Ein Leben wird besichtigt, Berlin, 2002, p. 233. 
fait nouveau, bien qu'il dérive de pratiques qui avaient déjà cours bien avant. Dès son enfance, Diederich Hesling, un enfant pleutre et fourbe qui sera un carriériste sans scrupule, est familier des «chants populaires» allemands sentimentaux. Non seulement il dénonce et fait punir un camarade d'école, mais il chante devant lui Le bon camarade ${ }^{22}$ (Der gute Kamerad). Ce chant de guerre triste, un poème composé en 1809 par Ludwig Uhland, était (et est encore de nos jours) interprété lors des obsèques des soldats ou des anciens combattants ou pour évoquer leur mémoire. La satire marque le traitement du chant dans le roman de Heinrich Mann. Au cours de ses études, Diederich apprend lors des rituels alcoolisés de sa corporation estudiantine le répertoire du Recueil général du chant de taverne allemand (Allgemeines Deutsches Kommersbuch), une anthologie composée par Friedrich Erk et Friedrich Silcher. Les auteurs avaient dédié en I858 la première édition de leur ouvrage à Ernst Moritz Arndt (I769-I860), d'une certaine manière le fondateur du nationalisme allemand. Diederich chante la «liberté» telle que l'imaginent ces étudiants, c'est-à-dire celle de boire, de maltraiter les "philistins" qui ne font pas d'études et de beugler des slogans nationalistes et impérialistes, au contraire des étudiants de 1848 qui composaient des chants républicains ${ }^{23}$. Le Sujet de l'Empereur cite plusieurs hymnes nationalistes, notamment La Garde sur le Rhin ${ }^{24}$, chant écrit en réaction à la crise du Rhin de 1840 . L'expression de "fidélité allemande" (deutsche Treue ${ }^{25}$ ), tirée du Chant des Allemands de Hoffmann von Fallersleben, est citée à plusieurs reprises ainsi que, dans un emploi ironique, la combinaison d'adjectifs "fidèle et bon" (treu und gut ${ }^{26}$ ) tirée du chant d'Ernst Moritz Arndt Qu'est la patrie de l'Allemand? (Was ist des Deutschen Vaterland?). Lors de l'inauguration de la statue de Guillaume $\mathrm{I}^{\text {er }}$ retentissent trois chants : le choral de Luther Notre Seigneur est une forteresse (Ein Feste Burg ${ }^{27}$ ), La Garde sur le Rhin (Die Wacht am Rhein) et l'hymne prussien Salut à toi, roi couronné du laurier des vainqueurs (Heil Dir im Siegerkranz $z^{28}$ ). À noter que l'on entend peut-être pour la première fois dans un roman allemand L'Internationale, entonnée par des sociaux-démocrates. Un anachronisme, le chant n'a été adapté en allemand

22. H. Mann, op. cit., p. I6 et suiv.

23. Ibid., p. 32 et 199 (chants d'étudiants) et 122 et suiv. et I3I et suiv. (chants républicains du "vieux" Buck). Dans Th. Mann, op. cit., p. I34, l'étudiant en médecine Morten Schwarzkopf inflige à la jolie Toni Buddenbrook des hymnes politiques qui ennuient «horriblement" (greulich) la jeune fille.

24. Ibid., p. 158, 264 (allusivement, le texte citant le mot Schwertgeklirr, cliquetis des épées), 370 et $47 \mathrm{I}$.

25. Ibid., p. 216, 348 .

26. Ibid., dès p. II.

27. Ibid., p. 465.

28. Le timbre est celui de l'hymne anglais God Save the King. Ibid., p. 47I. 
qu'en I9Io, soit plus d'une décennie après l'action" ${ }^{29}$. Si, sur le plan "public», le chant reflète les antagonismes sociaux et politiques, une ambiance de violence, sur le plan privé, Diederich Hessling recourt à un registre plus sentimental, surtout lorsqu'il ressent le besoin de compenser un choc ou une déception : il interprète alors des chansons folkloriques (Volkslieder) et joue sur son piano du Beethoven et du Schubert, parfois devant la chope de bière que lui a apportée sa mère compatissante : « $\mathrm{Ah}$, la bonne mère! Schubert, cœur probe et tendre, tendre génie de la patrie ${ }^{30}$."

Malgré le succès du roman de Heinrich Mann durant les premières années de la République de Weimar, la satire s'est révélée insuffisante. La fin, au plus haut point ambiguë, de Berlin Alexanderplatz d'Alfred Döblin (I929) suggère que l'heure est à une forme militarisée de militantisme, que l'on soit de gauche ou de droite. Le héros, Franz Biberkopf, citant plusieurs chants militaires, dont Le chant matinal du cavalier (Reiters Morgengesang ${ }^{31}$ ) de Wilhelm Hauff, dans une sorte de monologue intérieur, le roman se termine sur une note guerrière et semble appeler à la lutte finale des opprimés. L'absence d'analyse politique et la présence des slogans guerriers, mais creux, tirés de chants militants montrent que Biberkopf n'est pas capable de se doter d'un discours propre. Cependant, la narration indique une certaine prise de conscience du personnage principal, puisqu'il semble vouloir se prendre en main et désormais ne plus se contenter de vénérer le destin, mais «le regarder, le toucher et le détruire ${ }^{32}$ ». Jusqu'à cette ultime esquisse de prise de conscience, le personnage aura accompli un long et douloureux parcours, à travers le bruit du monde moderne, celui des tramways électriques, des automobiles, des orchestres, des enregistrements sonores diffusés et amplifiés partout, le bruit le plus obsédant étant l'écho encore vivant de la Première Guerre mondiale. Dans le perpétuel capharnaüm, les citations de chansons contribuent à créer une ambiance collective que l'on peut vivre de l'intérieur. Le lecteur, qui peine aujourd'hui à identifier des lambeaux de texte qui n'évoquent en lui aucune mélodie, repère toutefois les citations en fonction de critères stylistiques variés, tels que la rupture stylistique, le rythme, la rime, etc. Le roman d'Alfred Döblin puise dans deux registres, d'une part les hymnes politiques, de droite et de gauche, et les chants de soldats ${ }^{33}$, de l'autre les chansons de variétés et les opérettes

29. Ibid., p. 423. L'hymne ouvrier avait été adapté en allemand en I9ıo par Emil Luckhardt.

30. Ibid., p. 73 et 185 : "Die gute Mutter! Schubert, weiche Biederkeit, Gemüt der Heimat..."

3I. A. Döblin, op. cit., p. 4IO et suiv. Voir G. Sander, Alfred Döblin. Berlin Alexanderplatz. Erläuterungen und Dokumente, Stuttgart, Reclam, 1998, p. 4IO.

32. "[...] man muss es ansehen, anfassen und zerstören", ibid., p. 4IO.

33. Nous en avons dénombré quatorze, certains sont cités à plusieurs reprises. 
qui forment la partie la plus importante ${ }^{34}$. La "grande» musique a quasiment disparu dans un roman dont les protagonistes sont issus des bas-fonds de la société. La deuxième catégorie, "légère", où survivent quelques restes de chant populaire, par exemple Adieu, ma chère patrie (Nun ade, du mein lieb Heimatland ${ }^{35}$ ), permet de formuler des sentiments pour lesquels on ne dispose pas de langage personnel. La première catégorie, "publique», habille de mots le vécu communautaire, national, militaire et politique, des prises de position catégoriques et figées qui justifient un rapide recours à la violence collective. L'expérience fondamentale qui fonde cet «être-ensemble» inquiétant est celle de la Première Guerre mondiale. Ce conflit a traumatisé les hommes et renforcé les divisions politiques, au point qu'au lieu de dialoguer les adversaires politiques ("nationalistes» contre "communistes») se battent par hymnes interposés avant d'en venir aux mains. C'est en chantant La Garde sur le Rhin ${ }^{36}$ que Franz Biberkopf affronte, en sortant de prison, un Berlin métamorphosé par la modernité, en particulier par le tramway. Quand, dans une taverne, on l'invite à chanter, il entonne d'abord Le bon camarade $^{37}$ : les hommes, probablement tous des rescapés de la guerre, chantent avec lui cet hymne sentimental qu'ils n'ont eu que trop souvent l'occasion d'entendre. Lorsque Biberkopf enchaîne avec La Garde sur le Rhin, il se fait expulser de l'établissement aux accents de L'Internationale ${ }^{38}$. Biberkopf est un pacifique, il pense avoir tiré les leçons du crime qu'il a commis (il a tué sa maîtresse) et estime qu'il est possible de renoncer individuellement à la violence et de ne pas répéter ce que sa génération a vécu pendant la guerre. Peu avant l'épisode qui vient d'être rapporté, Franz Biberkopf cite, sans le nommer, un chant révolutionnaire pour tourner en dérision la violence que prônent les communistes : "Il faut que le sang des Princes coule à flots" ${ }^{39}$ ». Avant la prise de conscience finale, qui est celle de la nécessaire mobilisation politique, le héros de Berlin Alexanderplatz est hanté par le bruit de la Première Guerre

34. Nous avons dénombré deux allusions à la "grande» musique; trois airs d'opérette, cinq chansons enfantines et folkloriques et trente-deux succès commerciaux. À la même époque, dans sa version sarcastique de la tradition comédie populaire viennoise (Volksstück) Histoire de la forêt viennoise (Geschichten aus dem Wiener Wald, I93I) Ödön von Horváth a utilisé le même procédé de la citation musicale, aussi bien sur le plan du texte que de celui de l'accompagnement musical, pour dénoncer le langage tout-fait d'une petite bourgeoisie autrichienne dont la culture au plus haut point conventionnelle masque (mal) la barbarie.

35. A. Döblin, op. cit., p. 45.

36. Die Wacht am Rhein, ibid., p. I2. Ce chant est cité p. 49, 78, II3, 400, 403, 4IO.

37. Ibid., p. 77 et suiv.

38. Ce chant est aussi cité p. $243,359,380$.

39. Ibid., p. 73. Il s'agit d'une citation du Heckerlied, ce chant de la jeunesse républicaine de 1848 dans lequel on préconise de verser le sang des princes : "Fürstenblut muss fließen / Knüppelhageldick.» 
mondiale, mêlant souvenirs réels, lambeaux de chants patriotiques ("chère patrie, sois rassurée») ou allusions à des chants (le mythe des jeunes volontaires de Langemarck qui se seraient lancés en novembre I9I4 dans un assaut mortel en chantant Deutschland über alles) :

Et le roulement des trains, les canons tonnent, les grenades explosent, le barrage d'artillerie, le Chemin des Dames et Langemarck, chère patrie, sois rassurée, chère patrie, sois rassurée. Les abris dans les tranchées sont enfouis, les soldats y ont disparu. La mort roule son manteau, chante : Oh oui, oh oui ${ }^{40}$.

Près d'un quart de siècle plus tard le roman La Serre (1953) de Wolfgang Koeppen renoue avec les procédés mis en œuvre dans Berlin Alexanderplatz. L'action de ce roman, qui est considéré en Allemagne comme l'un des plus représentatifs de son époque, se déroule pendant deux jours tandis que se prépare le débat au Bundestag sur la communauté européenne de défense. Felix Keetenheuve, député d'un grand parti dans lequel on devine aisément le SPD, ancien émigré antinazi, vient de perdre sa jeune femme, qui a été poussée au suicide par sa solitude et les mauvaises fréquentations. Le roman tient, pour de longues parties, du monologue intérieur où se mêlent éléments de l'action et imagination. Au bout de deux jours et une nuit, confronté à son échec privé et public dans une Allemagne qu'il juge perdue car unanimement disposée à se réarmer, le héros du roman se jette dans le Rhin... geste commenté - ironiquement - de la phrase légèrement modifiée que prononce une vaillante Suissesse dans Guillaume Tell (1804) de Schiller : "C'est en sautant du pont qu'il trouva la libertét ${ }^{4}$ ». Ainsi résumé, le roman semble exprimer le point de vue "progressiste» de nombreux intellectuels européens dans les premières années de la guerre froide, et de fait un antiaméricanisme assez prononcé caractérise ce roman à plusieurs reprises. À y regarder de plus près, on est contraint de fortement nuancer ce constat. En réalité, le roman semble au moins aussi proche d'une solide tradition allemande du Kulturpessimismus que du progressisme des années 1950, et, sous cet angle, la critique change d'objet : la "droite» allemande est relativement mieux traitée que l'opposition $^{42}$ ou les généraux français et anglais qui se félicitent de l'amputation du

40. Ibid., p. 400 : "Und Rollen von Eisenbahnen, Kanonen krachen, Platzen der Handgranaten, Sperreuer, Chemin des dames und Langemarck, Lieb Vaterland magst ruhig sein, Lieb Vaterland magst ruhig sein. Die Unterstände verschüttet, hingesunken die Soldaten. Der Tod rollt seinen Mantel, singt : O ja, o ja." "Lieb, Vaterland... " cite le texte de La Garde sur le Rhin.

4I. Voir Fr. Schiller, Wilhelm Tell (I, 2, v. 329).

42. Curieusement, le personnage du «chancelier» (Adenauer) est traité avec une certaine déférence. En revanche, le chef de l'opposition, qui se nomme Knurrewahn, a droit à un portrait au vitriol. Le personnage est inspiré de Kurt Schumacher, une victime de la répression national-socialiste, qui refusa bruyamment après la guerre d'accepter les nouvelles frontières orientales de l'Allemagne. 
territoire allemand. En réalité, La Serre est un roman assez contradictoire, où cohabitent (assez mal) une volonté "moderniste» (Keetenheuve traduit Baudelaire et cite E. E. Cummings) et un discours de fond antimoderniste ${ }^{43}$ d'une part, la polémique et la mauvaise conscience de l'autre ${ }^{44}$. Il n'y a pas lieu ici d'étudier l'origine de ces contradictions, qui de toute évidence renvoient à l'histoire de l'Allemagne et de ses intellectuels, mais aussi à la biographie de l'auteur et à ses obsessions personnelles.

Le récit déploie une certaine polyphonie qui fait entendre les voix du personnage principal, lui-même dédoublé en homme «réel» et en «imagination", mais aussi les discours d'autres, voire la voix d'un narrateur-auteur, souvent ironique. Sur le plan du traitement de la musique, on retrouve un univers familier, Wagner, l'opérette, les chants militaires, le Volkslied, les chants religieux. Américanisation oblige, la variété moderne, anglo-saxonne ou allemande, est bien représentée. Le roman oppose le texte vertueux, soit la modernité de Baudelaire, de Verlaine et d'E. E. Cummings, les poètes traduits ou cités par Keetenheuve, ou celle du flot de conscience «joycien» omniprésent dans le roman, au tout-fait et tout-pensé vicieux du chant. Dès l'ouverture du roman, dans le "Nibelungenexpress» (nom fictif!) qui gagne la vallée du Rhin, on cite les filles du Rhin de l'opéra L'Or du Rhin de Wagner pour suggérer le bruit des rails : "Wagalaweia», un véritable leitmotiv du roman ${ }^{45}$. Le "wagnérisme» du roman confond l'approche ironique de Thomas Mann et la satire d'Heinrich Mann et permet de suggérer la violence et la permanence d'un kitsch morbide, mais menaçant propre à l'Allemagne. En revanche, le texte fait allusion, en un segment de phrase, à Luther et Schiller, pour ramener la religiosité des politiciens chrétien-démocrates à ses petites dimensions affairistes : "[...] le Seigneur n'était pas obligé d'être le chef des armées des anges logeant au-dessus de la voûte céleste ${ }^{46}{ }^{4}$. La première notion (en allemand Herr Zebaoth) cite le cantique de Luther "Notre Seigneur est une forteresse», la seconde l'«Ode à la joie» de Schiller, qui fut mis en musique par Beethoven dans le dernier mouvement de la IX ${ }^{e}$ symphonie.

43. Contre Le Corbusier, la variété moderne, la naissance de la société de consommation, voire l'homosexualité féminine (c'est une "tribade» alcoolique qui a causé la mort de la jeune femme aimée, elle-même fille de dirigeants nazis qui se sont suicidés) ou la présence de soldats noirs dans l'armée américaine (ils ont une "posture de jungle»).

44. Nous laissons ici de côté les pulsions "pédophilistes»(W. Koeppen, op. cit., p. I84) et l'impuissance de Keetenheuve face à l'action et à la création (il ne parvient même pas à terminer ses traductions), éléments centraux du roman quelque peu ignorés (ou tus?) par la critique.

45. Ibid., p. IO, I2, 23, I87. Le roman fait aussi allusion à Tannhäuser, p. I46 : «Holdes Mädchen (au lieu de: "Allmächtige Jungfrau...»).

46. Ibid., p. I55. 
Un emploi similaire est réservé à la chanson enfantine et au Volkslied, dont la naïveté, voire la bêtise paraissent pour le moins inappropriées dans le monde médiocre et sale que décrit le roman ${ }^{47}$. Même la lune n'est plus "bonne ${ }^{48}$ ", ce qui implique que le lecteur se souvienne du chant du XIx siècle Bonne lune, tu avances sans bruit (Guter Mond, du gehst so stille). Les chants religieux ne sont pas moins trompeurs, voire cyniques, puisque c'est une "tribade» qui entonne le chant du fiancé céleste d'après "La jeune nonne», poème mis en musique par Schubert ${ }^{49}$. Seules les citations du grinçant Opéra de quat' sous de Bertolt Brecht et Kurt Weill (1928) échappent à l'ironie et à la satire qu'elles recèlent elles-mêmes ${ }^{5 \circ}$.

Le roman cite une dizaine de chansons de variété allemandes et, à plusieurs reprises, la chanson Botch-a-me (version américanisée de Baccia mi) interprétée par Rosemary Clooney. Certaines citations, approximatives, rendent difficile l'identification des chansons. Ainsi, il est probable que la mouette qui va sur la plage de la mer du Nord est en réalité cette mouette qui se rend à Helgoland de la chanson nostalgique de 1934 Kleine Möwe, flieg nach Helgoland (texte de Bruno Balz) ${ }^{\text {s. }}$. Dans l'ensemble les citations de chansons de variété sont destinées à tourner en dérision les "grands" sentiments en vogue dans l'Allemagne du début des années I950, à commencer par la nostalgie des territoires perdus (auxquels il est fait indirectement référence une fois ${ }^{52}$ ) ou du Berlin non divisé d'avant $1945^{53}$. Le dernier "tube» cité est celui d'une chanteuse enfant (la petite Berlinoise Conny Froboess) qui symbolisa à l'époque le début du miracle économique et de loisirs retrouvés : N'oublie pas d'apporter ton maillot de bain (Pack die Badehose ein), un produit de l'industrie culturelle qui annonce sur un mode ironique le geste ultime du héros qui va se jeter dans le Rhin. Reste la propagande militaire et politique qui, selon le personnage central, continue de dominer les esprits : un visiteur du Bundestag cite, au début du roman, le chant des troupes d'assaut dont le texte précise qu'un lieutenant et dix hommes sont bien assez pour vaincre l'ennemi - et pour évacuer ce parlement qui n'est qu'un rassemblement de

47. C'est un chant "stupide" que Dansons, chantons et sautons (Lasset uns singen, tanzen und springen), ibid., p. 152.

48. Ibid., p. 152 .

49. Ibid., p. I86. Le texte est du de Jakob Nikolaus von Craigher de Jachelutta. Autres chants religieux : p. 138 et suiv.

50. Ibid., p. 95 et 177.

5I. Ibid., p. 172.

52. Ibid., p. 43.

53. Deux chansons de l'après-guerre sont citées, ibid., p. I43 et suiv. : Nostalgie du Kurfürstendamm (Heimweh nach dem Kurfürstendamm [musique de B. Kamp, texte de B. Kamp, G. Schwenn] et J'ai encore une valise à Berlin (Ich hab noch einen Koffer in Berlin [musique de R. M. Siegel, texte de A. von Pinelli]). 
bavards $s^{54}$, et dans le monologue délirant des derniers paragraphes, c'est la vision de troupes qui défilent en chantant l'hymne nazi (le Horst-Wessel-Lied), l'Internationale, La Marseillaise et La Carmagnole qui pousse Keetenheuve à trouver dans le Rhin une tombe. On sent ici que l'auteur s'est souvenu de la fin de Berlin Alexanderplatz et de son ambiguïté d'une certaine manière "antipolitique»: si l'on comprend aisément que l'ancien soldat Biberkopf ne soit pas parvenu à se doter d'un langage et d'une pensée réellement politiques, susceptibles de guider une action rationnelle, on comprend moins qu'un intellectuel aussi averti que le héros de La Serre confonde tous les discours, de la droite extrême à l'extrême gauche, en passant par le républicanisme français, dans la même aversion antimilitariste. D'un point de vue formel, on notera que l'aversion porte aussi sur l'industrie culturelle qui connait un essor accéléré à partir des années 1950 et du début de la conquête de ce secteur par le cinéma et la chanson anglo-saxons. Le roman rend compte de ces tendances tout en les combattant en manifestant un point de vue qui, loin d'être moderne, rappelle d'anciens préjugés culturels, moraux et ethniques. Ce point de vue montre qu'un intellectuel allemand, qui a vécu l'essentiel de sa vie de jeune adulte (de vingt-sept à trente-neuf ans) sous le Troisième Reich, peine à renouer avec l'avant-garde critique européenne et américaine, en particulier avec celle de la République de Weimar.

Nous avons réduit le champ de notre étude à quelques romans allemands particulièrement représentatifs des grandes tendances de l'histoire du genre au $\mathrm{xx}^{\mathrm{e}}$ siècle. Buddenbrooks décrivait, sur le plan musical, le passage d'un univers naiff et urbain à l'emphase nationale et militariste d'un côté et à une compensation wagnérienne impure et morbide de l'autre. Les nouveaux riches du Sujet de l'empereur d'Heinrich Mann trouvent dans la décadence wagnérienne l'occasion de confirmer leurs pires préjugés et ces affairistes incultes limitent leur goût musical aux hymnes officiels ou, aux heures sentimentales, au Volkslied et à une version sentimentale de Schubert et de Beethoven. Dans les bas-fonds berlinois, dont est issu Franz Biberkopf, c'est le souvenir du traumatisme infligé par la Première Guerre mondiale qui obsède les esprits, et le flot de musiques amplifiées et partout diffusées ne parvient pas à le submerger. Ces hommes trouvent cependant dans le tout-fait du texte industriel des chansons de variété et des opérettes un ersatz de pensée personnelle. Le fait qu'à la fin du roman une prise de conscience s'exprime par le truchement d'un discours évoquant le rythme des chants de marche

54. La citation «Un lieutenant et dix hommes» (Ein Leutnant und zehn Mann) est tirée du chant La troupe d'assaut (Der Stoßtrupp), ibid., p. 35 et I56. Il s'agit d'un chant de marche de la Première Guerre mondiale, en vogue encore dans la Wehrmacht (musique de H. Niel, texte de H. Anacker). 
incite à s'interroger sur sa qualité. Après la guerre, Wolfgang Koeppen tente de renouer dans La Serre avec cette réflexion sur la modernité et ses enjeux, mais les douze ans du Reich de mille ans ont laissé des traces, et le rejet des références "traditionnelles» (les hymnes militaires et religieux, Wagner) va de pair avec celui de la culture de masse et de tous les chants militants, quels qu'ils soient. Autrement dit, le monde des années 1950, qui furent la première décennie des «trente glorieuses», est présenté comme décadent, une analyse que confirme une étude de la place de la musique et du chant dans ce roman... et qu'infirment toutes les études historiques et économiques. Ce roman, qui en citant ironiquement des chants et des films ${ }^{55}$ moque l'omniprésence de la "nostalgie» (Heimweh) à cette époque, manifeste la nostalgie de l'époque où les masses (a fortiori les masses américanisées) n'avaient pas voix au chapitre.

Une autre conclusion, plus générale, s'impose. Dans le domaine des relations entre roman et chanson, l'intermédialité va de pair avec l'intertextualité : le roman cite sans indiquer ses sources, au lecteur d'identifier le collage, la variation, la citation, un travail "philologique» que l'archivage déficient de sources qui n'ont pas encore véritablement leur «science» et leurs "savants" rend ardu. Pourtant, une bonne connaissance de l'univers musical des Allemands est indispensable à qui veut comprendre les nombreux romans "qui connaissent la musique». Cette approche intermédiale peut s'étendre aisément à d'autres arts, à commencer par le thêâtre et le cinéma... et à d'autres pays.

55. Il est question d'un film qui aurait pour titre $\grave{A}$ Heidelberg au bord du Rhin sur la lande dans ma baignoire pour l'Allemagne sur le Drachenstein; p. 34. Il s'agit d'un pot pourri de titres réels et possibles de films et de chansons de cette époque riche en Heimatfilme, Heimatlieder, etc. 
